\title{
Universitätsklinikum Bonn (UKB) ist erstes Uniklinikum mit 5 DEGUM- zertifizierten Ausbildungszentren
}

Mit insgesamt 5 DEGUM-zertifizierten Ausbildungszentren ist das Universitätsklinikum Bonn (UKB) die Uniklinik mit den meisten DEGUM-zertifizierten Zentren in Deutschland. Die Zertifizierung steht für eine hervorragende Ausbildung der Assistenz- und Fachärzte. Außerdem fördert sie die wissenschaftliche Kooperation zwischen den DEGUM-zertifizierten Zentren untereinander und dem wissenschaftlichen Output auf dem Gebiet des Ultraschalls insgesamt.

Eine DEGUM-Zertifizierung bescheinigt neben der ausgezeichneten Ausbildungsqualität auch, dass moderne und hochwertige Ultraschallgeräte eingesetzt werden. Zu den Voraussetzungen gehört, dass die Abteilungen sowohl über Ausbilder in der Ultraschalldiagnostik verfügen als auch einen Nachweis für ein Ausbildungscurriculum sowie für bestimmte Geräte erbringen müssen. Das Zertifikat wird für 3 Jahre vergeben, danach besteht die Möglichkeit einer Rezertifizierung. Als Ultraschallzentrum kann eine Klinik zertifiziert werden, wenn es in ihrem Haus mindestens 3 DEGUM-zertifizierte Abteilungen und interdisziplinäre Zusammenarbeit (z. B. bei der US-Ausbildung) gibt. Außerdem müssen 1-mal pro Jahr gemeinsame Sonografie-Veranstaltungen durchgeführt werden. Durch die Zertifizierung wird zudem die wissenschaftliche Kooperation der DEGUM-zertifizierten Zentren in Deutschland untereinander intensiviert, dazu gehören gemeinsame Projekte und Publikationen.
„Erstmalig hat eine Uniklinik in Deutschland die DEGUM-Zertifizierung für 5 Kliniken erhalten“, erklärt Professor Dr. med. Christoph Berg, Leiter des Bereichs Fetalchirurgie der Universitätsfrauenklinik Bonn (DEGUM III) und Vorstandsmitglied der Fachgesellschaft. Am UKB wurden die Rheumatologie und klinische Immunologie der Medizinischen Klinik III, die Kinderkardiologie, die Geburtshilfe und pränatale Medizin, die Radiologie sowie die Angiologie der Medizinischen Klinik II zertifiziert.

\section{KRITERIEN FÜR \\ ZERTIFIZIERUNGEN}

Zertifizierung für qualifizierte Ultraschall-Weiterbildung für Abteilungen

Das Zertifikat kann beantragt werden von Abteilungen, die folgende Voraussetzungen erfüllen:

- DEGUM-Ausbilder (Stufe II) in der Abteilung

- Nachweis eines Ausbildungscurriculums

- Stellungnahme des Assistentensprechers zur Ultraschallweiterbildung (Ausbildungscurriculum wird praktisch umgesetzt)

- Gerätenachweis (DEGUM-Stufe II)

- Nachweis Literatur-/Lehrbuchfundus und Internetzugang

- die Abteilung/Einrichtung muss das gesamte Spektrum des jeweiligen Fachs abdecken

\section{Rezertifizierung}

Voraussetzungen für die

Rezertifizierung:

- Bestätigung DEGUM-Stufe II in der Abteilung

- Nachweis des aktuellen Ausbildungscurriculums

- Stellungnahme des Assistentensprechers zur Umsetzung des Curriculums

- Liste der ausgebildeten Kolleginnen und Kollegen in den letzten 3 Jahren

\section{Zertifizierung für interdisziplinäre} Ultraschallzentren

Das Zertifikat kann beantragt werden von Zentren, die folgende Voraussetzungen erfüllen:

- mindestens 3 DEGUM-zertifizierte Abteilungen an einem Haus

- interdisziplinäre Zusammenarbeit (z. B. bei der US-Ausbildung)

- Verwendung einer einheitlichen Terminologie; nach Möglichkeit einheitliche Befunderstellung

- Durchführung von gemeinsamen Sonografie-Veranstaltungen (wissenschaftliche Veranstaltung oder Fortbildung): mindestens eine Veranstaltung pro Jahr

- empfohlen: gemeinsame Seminare, Publikationen

- Audit

Anträge für die Zertifizierungen können unter www.degum.de/aktivitaeten/qua litaetssicherung/zertifizierungen.html gestellt werden. 